\title{
Contribuições de Agentes Comunitários de Saúde para a construção do perfil de usuários da Atenção Básica comnecessidades desaúdemental
}

\section{Community Health Agents contributions to set up the profile of Primary Care Services users with mental health demands}

\author{
Karyna Duarte Alcântara ${ }^{1}$ (D), Fernando Santos de Carvalho ${ }^{1}$ (D), Manuella Lima Belo (D), $^{\text {, }}$ \\ Maria Gerlane de Souto² (D), Adriana Marcia Emidio da Silva ${ }^{3}$ (D), Gabriela Andrade da Silva' (D) \\ 'Instituto de Humanidades, Artes e Ciências Jorge Amado da Universidade Federal do Sul da Bahia (UFSB) - Itabuna (BA), Brasil. \\ ${ }^{2}$ Secretaria Municipal de Saúde de Itabuna, Núcleo de Planejamento, Informação e Gestão Estratégica da Saúde - Itabuna (BA), Brasil. \\ ${ }^{3}$ União Metropolitana de Educação e Cultura (Unime) - Itabuna (BA), Brasil.
}

Como citar: Alcântara KD, Carvalho FS, Belo ML, Souto MG, Silva AME, Silva GA. Contribuições de Agentes Comunitários de Saúde para a construção do perfil de usuários da Atenção Básica com necessidades de saúde mental. Cad Saúde Colet, 2020;28(4):599-608. https://doi.org/10.1590/1414-462X202028040014

\section{Resumo}

Introdução: A Atenção Básica à Saúde, que integra a Rede de Atenção Psicossocial (RAPS), deve apresentar papel fundamental para a identificação de casos, acompanhamento e promoção da saúde mental, ações com as quais os Agentes Comunitários de Saúde (ACS) podem contribuir. Objetivo: Analisar a situação de saúde mental do município de Itabuna-BA, comparando informações fornecidas por ACS e pelo Sistema de Informação em Saúde para a Atenção Básica (SISAB). Método: Trata-se de pesquisa exploratória, descritiva, documental, que recuperou informações do SISAB e de Fichas de Cadastro da Coordenação de Saúde Mental, preenchidas por ACS. Resultados: A participação dos ACS elevou em mais de seis vezes o número de pessoas com transtornos mentais identificadas, mas o número de usuários de álcool e outras substâncias foi inferior ao registrado no SISAB. Foram constatadas evidências de desarticulação da RAPS e modelo de atenção biologizante e hospitalocêntrico. Conclusão: As fontes de informação se complementaram, evidenciando subnotificação dos casos de saúde mental e bom potencial dos ACS para auxiliar na busca ativa de usuários com transtorno mental, mas não de usuários de substâncias. Problemas de saúde mental relacionaram-se com aspectos de vulnerabilidade socioeconômica, indicando a necessidade de articulação intersetorial para oferecer atenção integral à saúde.

Palavras-chave: saúde mental; agentes comunitários de saúde; epidemiologia; atenção primária à saúde; sistemas de informação em saúde.

\begin{abstract}
Background: Primary Care Services is part of the Brazilian Psychosocial Care Network (PCN) and is expected to play a fundamental role in the identification of cases, monitoring and promotion of mental health, to which Community Health Agents (CHA) can contribute. Objective: Analyze the mental health situation of the municipality of Itabuna - BA, compare information provided by $\mathrm{CHAs}$ and the Health Information System for Primary Care (SISAB). Method: This was an exploratory, descriptive, documentary research that retrieved information from the SISAB and from Mental Health Coordination registry files, completed by CHAs. Results: The participation of CHAs increased by more than six times the number of people with
\end{abstract}

Trabalho realizado no Instituto de Humanidades, Artes e Ciências Jorge Amado, Universidade Federal do Sul da Bahia (UFSB) - Itabuna (BA), Brasil.

Correspondência: Gabriela Andrade da Silva. E-mail: gabriela.silva@ufsb.edu.br

Fonte de financiamento: Trabalho desenvolvido como parte das atividades do Programa de Educação pelo Trabalho para a Saúde - PET Saúde/GRADUASUS da Secretaria de Gestão do Trabalho e da Educação na Saúde (SGTES) do Ministério da Saúde (MS), Brasil.

Conflito de interesses: nada a declarar.

Recebido em: Fev. 20, 2019. Aceito em: Out. 20, 2019 
mental disorders identified, but the number of users of alcohol and other drugs was lower than that registered in SISAB. Conclusion: There was evidence of PCN disarticulation and a model of biological and hospital-centered care. The sources of information complemented each other, evidencing underreport of mental health cases and good potential for $\mathrm{CHAs}$ assistance in the active search of users with mental disorders, but not of drug users. Mental health problems were related to aspects of socioeconomic vulnerability, indicating the need for intersectoral articulation to provide comprehensive health care.

Keywords: mental health; community health agents; epidemiology; primary health care; health information systems.

\section{INTRODUÇÃO}

O presente artigo trata de uma experiência de participação de Agentes Comunitários de Saúde (ACS) na identificação e construção do perfil de Pessoas com Necessidades de Saúde Mental (PNSM) no território, subsidiando o planejamento das ações da Atenção Básica à Saúde no âmbito da Rede de Atenção Psicossocial (RAPS). Concebida no âmbito da Reforma Psiquiátrica brasileira, a RAPS ${ }^{1}$ redirecionou o modelo assistencial em saúde mental, ao criar no Sistema Único de Saúde (SUS) uma rede articulada de pontos de atenção para pessoas com sofrimento ou transtorno mental e com necessidades decorrentes do uso de substâncias.

Embora os Centros de Atenção Psicossocial (CAPS) sejam frequentemente compreendidos como dispositivos centrais, estratégicos e organizadores da RAPS², a Atenção Básica (AB) é a ordenadora das redes de atenção à saúde, de acordo com a Política Nacional de Atenção Básica $(\mathrm{PNAB})^{3}$. Mais do que simples porta de entrada, tem o papel de acolhimento e acompanhamento integral e longitudinal dos usuários, elaborando, acompanhando e gerindo o projeto terapêutico singular e articulando o fluxo nos demais dispositivos ${ }^{3}$. Por sua capilaridade nos territórios, serviços de $A B$ ocupam posição privilegiada na RAPS para o acolhimento e conhecimento das pessoas, famílias, históricos de vida, vínculos e redes de apoio. Nesse contexto, os ACS atuam como elo entre serviço de saúde e comunidade, pois moram no território onde atuam 4 .

Pesquisas com amostras probabilísticas de base populacional encontraram prevalências em torno de $30 \%$ para transtornos mentais comuns em Feira de Santana-BA ${ }^{5}$ e Olinda-PE 6 . Embora altamente prevalentes, transtornos mentais ficam invisíveis na formulação das políticas públicas brasileiras por causa de subnotificação. Um levantamento dos dados do SIAB (antigo Sistema de Informação da Atenção Básica) constatou que menos de $1 \%$ dos municípios registrou distúrbios mentais e que os registros corresponderam a pouco mais de $0,5 \%$ da estimativa de prevalência de transtornos mentais graves e persistentes ${ }^{7}$.

Investigações de determinantes sociais em estudos epidemiológicos sobre transtornos mentais são praticamente consensuais em afirmar que há relação entre fatores socioeconômicos e transtornos mentais comuns, embora variem quanto aos tipos de associação encontrados e modelos explicativos ${ }^{8}$. Um estudo qualitativo brasileiro traz contribuição relevante ao analisar a experiência de implantação de uma ouvidoria em comunidades em situação de extrema pobreza, nas quais o sofrimento psíquico difuso é endêmico. Conceituando pobreza como não satisfação adequada das necessidades, sua definição não se limita à indisponibilidade do mínimo indispensável para a sobrevivência física, pois é determinada pela definição socialmente construída do que é necessidade; portanto, é apresentada em relação indissolúvel com a desigualdade social. Na escuta de representantes das comunidades - entre os quais um ACS -, foram expostas dimensões subjetivas decorrentes da precariedade socioeconômica, referentes aos desejos, medos e sofrimentos amplificados pela disseminação da cultura do "ter". As dificuldades no acesso a cuidados em saúde agravam o adoecimento9.

Os ACS, enquanto membros de suas comunidades, estão expostos às mesmas redes de determinação em saúde e ocupam espaço privilegiado para a construção de conhecimentos sobre a saúde mental no território

[...] dinâmico, adscrito, permeado pelo compartilhamento de valores, crenças, práticas e formas intensamente ativas de relacionamentos, [que] constitui local de veemente produção de sentidos acerca da vida que circula ali ${ }^{10: 113}$. 
Quantitativamente, isso se verificou em experiência realizada em uma unidade de saúde da família de Cuiabá- MT, onde o número de usuários com transtornos mentais identificados subiu de 19, conforme o SIAB, para 39, após busca ativa por ACS capacitados ${ }^{11}$.

Entretanto, ACS e outros profissionais da AB podem apresentar sentimento de impotência no acompanhamento de usuários no âmbito da saúde mental, apontando limitações como deficiência na formação, ausência de rede articulada, baixa disponibilidade de recursos e, consequentemente, insuficiência das ações na $A B$, persistindo práticas medicocêntricas e manicomiais, sendo os medicamentos e a internação as principais abordagens assistenciais ${ }^{12-14}$. somado a isso o medo da loucura, a qual, no imaginário coletivo, é vinculada à periculosidade ${ }^{10,15}$.

Esse temor se acentua na relação com o uso de substâncias psicoativas (SPA), dadas as representações sociais que o associam com criminalidade e perigo, marcadas por juízos de valor que desqualificam e marginalizam os usuários ${ }^{15}$. Inseridos nesse mesmo universo social, ACS e demais membros da equipe de saúde da família tendem a apresentar estigmatização e concepções reducionistas potencializadas por modelos de atenção proibicionistas, oferecendo modelos de cuidado inefetivos, distanciados do princípio de integralidade $\mathrm{e}^{16-18}$ e do paradigma da redução de danos que norteia as políticas públicas de saúde ${ }^{19}$.

Apesar dessas limitações, os ACS, "No cuidado em SM, [...] desenvolvem habilidades no uso de tecnologias baseadas especialmente na relação e aprendidas no cotidiano de visitas, casa a casa"10:116. Aprendidas e evocadas durante a produção do cuidado, essas tecnologias "[...] vão além dos medicamentos, equipamentos e procedimentos usados na assistência à saúde"10:116.

O apoio matricial pode empoderar os ACS para identificar e atuar no acompanhamento de PNSM em seus territórios, empregando efetivamente tecnologias leves, como escuta, acolhimento e vínculo, construindo elos entre usuários, famílias, comunidade e unidade de saúde e contribuindo, portanto, para a integralidade das ações ${ }^{20-24}$. O apoio matricial por CAPS ou Núcleo Ampliado de Saúde da Família e Atenção Básica (Nasf-AB) - embora inexistente em diversos municípios - pressupõe responsabilidade sobre os usuários compartilhada pelas equipes de $A B$ e atenção especializada, de forma horizontal, não hierarquizada, envolvendo colaboração no estabelecimento de diagnóstico, construção conjunta de projetos terapêuticos singulares e intervenções ${ }^{1,3}$.

A presente pesquisa foi realizada em Itabuna, localizado no sul do estado da Bahia, município com 204.667 habitantes em 2010²5. Em 2016, havia 32 Unidades Básicas de Saúde, um CAPS II, um CAPS Infantil, um CAPS Álcool e Drogas e leitos psiquiátricos em hospital geral. Em 2015, os ACS participaram do projeto "Caminhos do Cuidado", realizado por uma parceria entre o Ministério da Saúde, o Grupo Hospitalar Conceição e a Fundação Oswaldo Cruz (Fiocruz), que promoveu capacitação quanto aos cuidados a usuários com transtornos mentais, especialmente o uso de crack, álcool e outras substâncias, e articulação entre as proposições das políticas de saúde mental e de $A B^{26}$. Nessa oportunidade, a Coordenação de Saúde Mental do município solicitou que os ACS identificassem PNSM em seus territórios, preenchendo uma ficha de cadastro.

O objetivo da presente pesquisa foi analisar quantidade, perfil e situação de saúde de usuários da $A B$ com necessidades de saúde mental, comparando informações fornecidas pelos ACS com dados presentes no SISAB. A pretensão, assim, era contribuir para a avaliação do impacto da participação de ACS na identificação de usuários com transtornos mentais e descrição de seu perfil, além de subsidiar, em nível local, o planejamento de ações na área de saúde mental na $A B$ do município.

\section{MÉTODO}

Trata-se de estudo descritivo exploratório, com abordagem quantitativa e documental, aprovado pelo Comitê de Ética em Pesquisas com Seres Humanos da Fundação Pública de Saúde de Vitória da Conquista, CAAE: 63289816.2.0000.8089.

Foram usadas duas fontes de informação: 1. Sistema de Informação em Saúde para a Atenção Básica (SISAB/e-SUS): é uma das estratégias do Ministério da Saúde para desenvolver, reestruturar e integrar os sistemas de informação, permitindo registro individualizado da 
situação de saúde por meio do Cartão Nacional de Saúde (Cartão SUS) ${ }^{27,28}$. Os dados, referentes ao período de janeiro a dezembro de 2016, foram recuperados de forma agregada dos sistemas de informação e analisados por meio de planilhas no software Microsoft Excel; 2. Fichas de cadastro preenchidas por ACS referentes às PNSM identificadas no segundo semestre de 2015, com levantamento de dados sociodemográficos e de saúde. Os dados foram analisados estatisticamente de forma descritiva usando o software IBM SPSS 20.0.

\section{RESULTADOS}

\section{Dados do SISAB}

No período analisado, existiam em Itabuna 32 unidades de saúde físicas, nas quais atuavam 44 equipes de AB. Dessas equipes, 24 operavam na modalidade de Estratégia Saúde da Família ${ }^{2}$ (cobertura de 29,3\%), e 20, na modalidade Equipes de Agentes Comunitários de Saúde 3 .

O cadastro do e-SUS ${ }^{4}$ continha 107.519 usuários, dos quais $54,6 \%$ eram do sexo feminino. A escolaridade máxima de $50,5 \%$ foi ensino fundamental; $26 \%$ tinham ensino médio; $8,3 \%$ possuíam ensino superior; outros 7,5\% nunca frequentaram instituições de ensino; e os demais dados se referem à creche, pré-escola e cursos de alfabetização para adultos. Quanto à situação laboral, 14,4\% eram aposentados ou pensionistas, 9,8\% eram desempregados e $32,2 \%$ não trabalhavam.

Foram informadas 82 pessoas $(0,08 \%)$ com problema de saúde mental, $13.350(12,4 \%)$ consumiam álcool e $772(0,7 \%)$ faziam uso de outras substâncias. Foram registrados 2.054 $(2,19 \%)$ atendimentos de saúde mental, $174(0,19 \%)$ de usuários de álcool e $88(0,09 \%)$ de usuários de outras substâncias por profissionais de saúde da $A B$, de um total de 93.916 atendimentos ${ }^{5}$. Os ACS, por sua vez, realizaram $3.362(1,90 \%)$ visitas domiciliares por motivo de saúde mental, 4.457 (2,52\%) para usuários de álcool e 416 (0,24\%) para usuários de outras substâncias ${ }^{6}$, de um total de 176.952 visitas. Foram registrados 55 encaminhamentos para CAPS.

\section{Dados das fichas de cadastro preenchidas pelos ACS}

Foram retornadas as fichas de cadastro de 597 pessoas, cujo perfil sociodemográfico é apresentado na Tabela 1. Houve predominância de pessoas do sexo masculino (53\%), com faixa etária de 31 a 60 anos (60,5\%), baixa escolaridade (76,4\% tinham, no máximo, ensino fundamental completo), que não trabalhavam $(91,9 \%)$ e recebiam auxílios socioeconômicos $(66,6 \%)$.

Quanto ao perfil clínico (Tabela 2), 85,6\% foram identificados com transtorno mental, e 16,6\%, como usuários de álcool e outras substâncias (item permitia respostas múltiplas). Mais da metade apresentava sintomatologia referente a transtorno mental há mais de 10 anos $(58,2 \%)$ e algum familiar com transtorno mental $(51,2 \%)$. Foram verificadas omissões no preenchimento do item sobre uso de SPA em $76,9 \%$ das fichas.

Sobre a assistência à saúde mental, conforme descrito na Tabela 3, 77,3\% usavam medicamentos, 41,8\% já usaram serviços de urgência ou emergência (Serviço Móvel de Atendimento de Urgência ou hospital geral), 59,4\% passaram por internação e 40,8\% das internações ocorreram em hospital psiquiátrico. Serviços de média complexidade (ambulatório, (APS) foram acionados com menor frequência.

\footnotetext{
Dados do Ministério da Saúde, Departamento de Atenção Básica, e-SUS AB, base local da Secretaria Municipal de Itabuna-BA.

2 Equipe completa, de acordo com a Política Nacional de Atenção Básica.

3 Equipe composta de 1 enfermeiro e de 4 a 12 ACS.

4 Os resultados apresentados se referem aos casos válidos. A escolaridade e a situação laboral não foram informadas em $15,3 \%$ e $31,3 \%$ do total de registros, respectivamente.

5 Os atendimentos podem ter sido do mesmo usuário. Outros 17.692 atendimentos não identificaram a condição avaliada.

6 As visitas podem ter sido para o mesmo usuário. Outras 282.528 visitas não tiveram motivo identificado.
} 
Tabela 1. Perfil sociodemográfico de usuários da Atenção Básica com problemas de saúde mental identificados pelos Agentes Comunitários de Saúde (ACS), Itabuna-BA, 2016

\begin{tabular}{|c|c|c|}
\hline Variáveli & Frequência absoluta & Frequência relativa (\%) \\
\hline \multicolumn{3}{|l|}{ Sexo $(N=594)$} \\
\hline Masculino & 310 & 52,2 \\
\hline Feminino & 284 & 47,8 \\
\hline \multicolumn{3}{|l|}{ Faixa etária ( $\mathrm{N}=565)$} \\
\hline $0-18$ & 69 & 12,2 \\
\hline $19-30$ & 89 & 15,8 \\
\hline $31-45$ & 174 & 30,8 \\
\hline $46-60$ & 168 & 29,7 \\
\hline 60 ou mais & 65 & 11,5 \\
\hline \multicolumn{3}{|l|}{ Escolaridade $(\mathrm{N}=444)$} \\
\hline Analfabeto & 125 & 28,2 \\
\hline Fundamental incompleto & 131 & 29,5 \\
\hline Fundamental completo & 83 & 18,7 \\
\hline Médio incompleto & 47 & 10,6 \\
\hline Médio completo & 56 & 12,6 \\
\hline Superior completo & 2 & 0,5 \\
\hline \multicolumn{3}{|l|}{ Ocupação: trabalha? (N = 572) } \\
\hline Sim & 46 & 8,1 \\
\hline Não & 524 & 91,9 \\
\hline \multicolumn{3}{|c|}{ Recebe aposentadoria, benefício, auxílio socioeconômico?ii (N = 536) } \\
\hline Sim & 357 & 66,6 \\
\hline Aposentadoria / INSS / "Encostado" & 146 & 27,3 \\
\hline BPC / LOAS / "Amparo social" & 26 & 4,9 \\
\hline Bolsa Família & 20 & 3,7 \\
\hline Pensão / "Herança” & 7 & 1,3 \\
\hline Não indicado & 41 & 7,6 \\
\hline Não & 179 & 33,4 \\
\hline
\end{tabular}

' Número de casos válidos de cada variável indicado entre parênteses. ii Pergunta aberta. Os auxílios indicados pelos ACS foram agrupados em uma só categoria quando havia diferentes expressões para o mesmo benefício.

\section{DISCUSSÃO}

A participação dos ACS ampliou mais de seis vezes o número de registros do SISAB, corroborando estudo anterior ${ }^{11}$ e revelando seu potencial para apoiar a identificação de usuários com transtornos mentais no território. No entanto, sua contribuição não foi satisfatória para a identificação de pessoas usuárias de SPA: os ACS declararam 88 usuários de álcool e 14 de outras substâncias, enquanto o SISAB registrou 13.350 e 772, respectivamente. O item que identificava uso de SPA estava em branco em mais de $3 / 4$ das fichas retornadas. Conforme relatos informais, essa omissão se relacionou à apreensão em relação a possíveis ameaças e 
Tabela 2. Perfil clínico de usuários da Atenção Básica com problemas de saúde mental identificados pelos Agentes Comunitários de Saúde (ACS), Itabuna-BA, 2016

\begin{tabular}{|c|c|c|}
\hline Variáveli & Frequência absoluta & Frequência relativa (\%) \\
\hline \multicolumn{3}{|l|}{ Condição identificada } \\
\hline Transtorno mental $(\mathrm{N}=591)$ & 510 & 85,6 \\
\hline Álcool e outras substâncias ( $\mathrm{N}=590)$ & 99 & 16,6 \\
\hline Adesão ao tratamento $(\mathrm{N}=445)$ & 347 & 78,9 \\
\hline \multicolumn{3}{|l|}{ Doenças pregressas } \\
\hline Diabetes $(\mathrm{N}=586)$ & 41 & 7 \\
\hline Hipertensão arterial sistêmica ( $\mathrm{N}=585$ ) & 113 & 19,3 \\
\hline Cardiopatias $(\mathrm{N}=585)$ & 25 & 4,3 \\
\hline Outras $(\mathrm{N}=586)$ & 59 & 10,1 \\
\hline \multicolumn{3}{|c|}{ Apresenta histórico familiar de transtorno mental } \\
\hline $\operatorname{Sim}(N=590)$ & 308 & 51,2 \\
\hline Pais $(\mathrm{N}=588)$ & 105 & 17,9 \\
\hline Irmãos ( $\mathrm{N}=590)$ & 122 & 20,3 \\
\hline Tios $(\mathrm{N}=590)$ & 87 & 14,7 \\
\hline Avós paternos $(\mathrm{N}=590)$ & 24 & 4,1 \\
\hline Avós maternos ( $\mathrm{N}=589)$ & 26 & 4,4 \\
\hline Primos $(N=590)$ & 78 & 13,2 \\
\hline Outros $(\mathrm{N}=590)^{\mathrm{ii}}$ & 23 & 4,9 \\
\hline \multicolumn{3}{|l|}{ Sintomas identificados } \\
\hline Isolamento ( $\mathrm{N}=589)$ & 205 & 34,8 \\
\hline Apatia $(\mathrm{N}=590)$ & 105 & 17,8 \\
\hline Insônia (N = 590) & 300 & 50,8 \\
\hline Ansiedade $(\mathrm{N}=590)$ & 345 & 58,5 \\
\hline Alucinações ( $N=590)$ & 218 & 36,9 \\
\hline Delírios ( $\mathrm{N}=590)$ & 172 & 29,2 \\
\hline Agitação ( $N=590)$ & 403 & 68,3 \\
\hline Sintomas depressivos $(\mathrm{N}=590)$ & 246 & 41,7 \\
\hline Agressividade $(\mathrm{N}=590)$ & 265 & 44,9 \\
\hline Dificuldade de aprendizagem $(\mathrm{N}=590)$ & 266 & 45,1 \\
\hline Déficit de atenção ( $N=590)$ & 199 & 33,7 \\
\hline Substâncias psicoativas ( $N=590)$ & 29 & 4,9 \\
\hline \multicolumn{3}{|l|}{ Tempo do sintoma $(N=531)$} \\
\hline 0 a 2 anos & 55 & 10,4 \\
\hline 3 a 5 anos & 92 & 17,3 \\
\hline 6 a 9 anos & 75 & 14,1 \\
\hline 10 anos ou mais & 309 & 58,2 \\
\hline \multicolumn{3}{|l|}{ Hábitos de consumo de SPA } \\
\hline Álcool ( $N=431)$ & 88 & 20,4 \\
\hline Tabaco (N = 426) & 101 & 23,7 \\
\hline Outras SPA (N = 139) & 14 & 10,1 \\
\hline
\end{tabular}

i Número de casos válidos de cada variável indicado entre parênteses. ii Mencionados: bisavó, filho, sobrinho e esposa. Embora não configurem histórico familiar, informam presença de outras PNSM na mesma família, que podem onerar o cuidador. 
Tabela 3. Características da assistência à saúde mental de usuários da Atenção Básica identificados pelos Agentes Comunitários de Saúde (ACS), Itabuna-BA, 2016

\begin{tabular}{|c|c|c|}
\hline Variáveli & Frequência absoluta & $\begin{array}{l}\text { Frequência relativa } \\
(\%)\end{array}$ \\
\hline \multicolumn{3}{|l|}{ Atendimentos anteriores em saúde mental $(\mathbf{N}=\mathbf{5 8 8})$} \\
\hline Psicológico & 107 & 18,2 \\
\hline Psiquiátrico & 298 & 50,7 \\
\hline \multicolumn{3}{|l|}{ Uso de serviços } \\
\hline Ambulatório ( $\mathrm{N}=588)$ & 144 & 24,5 \\
\hline CAPS $(\mathrm{N}=588)$ & 200 & 34 \\
\hline Assistência social: CRAS/CREAS ( $\mathrm{N}=588)$ & 20 & 3,4 \\
\hline Centro de recuperação para usuários de SPA ( $\mathrm{N}=585$ ) & 48 & 8,2 \\
\hline Urgência / Emergência ( $N$ = 505) & 211 & 41,8 \\
\hline \multicolumn{3}{|l|}{ Número de internações ( $N=515)$} \\
\hline Nenhuma & 242 & 47 \\
\hline 1 a 4 vezes & 191 & 37,1 \\
\hline 5 a 9 vezes & 31 & 6 \\
\hline Mais de 10 vezes & 51 & 9,9 \\
\hline \multicolumn{3}{|l|}{ Local da última internação $(\mathrm{N}=331)$} \\
\hline Hospital geral & 100 & 30,2 \\
\hline Hospital psiquiátrico & 135 & 40,8 \\
\hline Uso de medicação ( $N=594)$ & 459 & 77,3 \\
\hline
\end{tabular}

' Número de casos válidos de cada variável indicado entre parênteses.

retaliações de usuários e traficantes, ratificando a representação social de periculosidade de usuários de SPA dominante no imaginário social| ${ }^{15-18}$.

A prevalência de transtornos mentais, mesmo após ampliada pela participação dos ACS, foi inferior ao esperado de acordo com estudos de base populacional, que apontaram taxas em torno de $30 \%$ para transtornos mentais comuns em cidades de grande porte do interior da região Nordeste ${ }^{5,6}$. O SISAB registrou apenas $0,8 \%$, concordando com o trabalho de Souza ${ }^{7}$ quanto à subnotificação.

É possível inferir limitações na participação dos ACS na identificação de PNSM, por exemplo: baixa motivação, dificuldade de identificação de transtornos mentais, tabus e estigmas relacionados ao tema ${ }^{10}$, representações sociais de periculosidade, sobretudo quanto ao uso de $S P A^{15}$ e déficits de trabalho em equipe e interprofissionalidade na AB. Algumas características clínicas levam a inferir que os ACS tenderam a identificar os casos mais graves: mais da metade passou por internação e apresentava sintomas há mais de 10 anos; agressividade foi relatada em $45 \%$, confirmando o estereótipo de que loucos são perigosos. Provavelmente, casos moderados e leves permaneceram ignorados.

Ainda assim, as informações retornadas pelos ACS possibilitaram descrever um perfil de pessoas com problemas de saúde mental no município, o que se assemelhou ao encontrado em Olinda ${ }^{6}$ : predomínio de adultos com baixa escolaridade, em idade economicamente ativa e que não trabalhavam. Ou seja, a vulnerabilidade social foi comum entre pessoas com problemas de saúde mental em ambas as cidades nordestinas e remete à patente relação entre pobreza, desigualdade social e sofrimento psíquico ${ }^{8,9}$. 
O perfil construído revelou proporção de pessoas que não trabalhavam $(91,9 \%)$ e analfabetas $(28,2 \%)$ superior à população geral: o SISAB registrou $56,4 \%$ pessoas sem ocupação no território e 7,5\% de pessoas que nunca frequentaram instituições de ensino; a taxa de analfabetismo no município de Itabuna foi de 10,9\%, conforme o IBGE ${ }^{24}$. Ficaram evidentes, portanto, a exclusão das PNSM de atividades sociais e produtivas - embora não seja possível estabelecer se foi como causa ou efeito dos problemas de saúde mental-e, consequentemente, o impacto econômico para todo o sistema de proteção social, incluindo saúde, previdência (aposentadorias por invalidez) e assistência social (Benefício de Prestação Continuada - BPC / Lei Orgânica de Assistência Social - LOAS).

Além das dificuldades socioeconômicas, parte dos usuários apresentou doenças físicas que demandavam acompanhamento pela $A B$, cronificação do quadro de saúde mental e presença de outras pessoas da família com problemas de saúde mental, tendendo a sobrecarregar cuidadores. Portanto, foram agregados fatores biopsicossociais de vulnerabilidade, demandando ações intersetoriais, viabilizadas pela clínica ampliada, compartilhada, voltada à integralidade do cuidado, e acompanhamento dos ACS por meio de tecnologias leves, tais como a escuta qualificada e a orientação familiar ${ }^{10,20-24}$.

Entretanto, a frequência de atendimentos de saúde mental na $A B$ e de encaminhamentos para serviços especializados foi baixa. Serviços de média complexidade, como CAPS e ambulatórios, foram pouco frequentes, mas foi comum a internação psiquiátrica, situando Itabuna na contramão da Reforma Psiquiátrica e da orientação da Política Nacional de Saúde Mental. Ainda, o acesso a atendimento psiquiátrico foi mais frequente que ao psicológico, e o tratamento mais relatado foi medicamentoso, indicando priorização dos aspectos biológicos dos transtornos mentais em detrimento de intervenções psicossociais. Essas informações podem ser compreendidas à luz do contexto local, pois em Itabuna havia um hospital psiquiátrico que encerrou suas atividades em 2015 de forma abrupta, sem construção de rede substitutiva para a desinstitucionalização.

Nesse contexto, não surpreende que os resultados tenham sinalizado severas limitações da rede substitutiva no município de Itabuna. Cabe acrescentar que, no período em que foi realizada a coleta de dados, o município não dispunha de matriciamento. Um Nasf-AB foi implantado posteriormente, em 2017, mas cobria apenas parcialmente a rede de AB. É fundamental investir na ampliação e articulação da RAPS e assegurar o matriciamento por meio dos Nasf-AB ou CAPS, proporcionando às equipes de AB recursos para lidar com os problemas de saúde mental, altamente prevalentes no território.

A presente pesquisa contribuiu para a discussão da possibilidade de incluir ACS, devidamente capacitados e com apoio matricial, na identificação e construção do perfil de PNSM no território e enfrentamento da subnotificação. A identificação de usuários de SPA, no entanto, demandaria maior cuidado na educação permanente para desconstrução de estigmas. As duas fontes de informação (SISAB e cadastro realizado por ACS) revelaram informações complementares.

Aspectos socioeconômicos desfavoráveis apresentaram relação com quadros de transtorno mental, indicando necessidade de articulação intersetorial, na perspectiva de atenção integral à saúde, bem como desarticulação da rede para atender usuários de saúde mental, potencializada pela ausência de apoio matricial. A despeito dos esforços que têm sido empreendidos, em âmbito nacional, para adequação aos princípios da Reforma Psiquiátrica, o paradigma medicocêntrico e biologizante predominou no município de Itabuna. As principais recomendações para a gestão, portanto, são ampliar o número de dispositivos da RAPS e proporcionar apoio matricial e educação permanente para as equipes, incluindo ACS, empoderando-os para os cuidados a $\mathrm{PNSM}^{20-24}$ e possibilitando que a $\mathrm{AB}$ assuma, efetivamente, seu papel de ordenadora dos cuidados em saúde 3 .

\section{REFERÊNCIAS}

1. Brasil. Ministério da Saúde. Portaria no. 3.088, de 23 de dezembro de 2011. Institui a Rede de Atenção Psicossocial para pessoas com sofrimento ou transtorno mental e com necessidades decorrentes do uso de crack, álcool e outras drogas, no âmbito do Sistema Único de Saúde (SUS). Diário Oficial da União, 26 dez 2011; Seção 1. 
2. Nunes MO, Onocko-Campos R. Prevenção, atenção e controle em saúde mental. In Paim JS, Almeida-Filho NM. Saúde coletiva: teoria e prática. Rio de Janeiro: Medbook; 2014. p. 501-512.

3. Brasil. Ministério da Saúde. Portaria nº. 2.436, de 21 de setembro de 2017. Aprova a Política Nacional de Atenção Básica, estabelecendo a revisão de diretrizes para a organização da Atenção Básica, no âmbito do Sistema Único de Saúde (SUS). Diário Oficial da União, Brasília, 22 de setembro de 2017; Seção 1.

4. Castro TA, Davoglio RS, Nascimento AAJ, Santos KJS, Coelho GMP, Lima KSB. Agentes Comunitários de Saúde: perfil sociodemográfico, emprego e satisfação com o trabalho em um município do semiárido baiano. Cad Saude Colet. 2017;25(3):294-301. http://dx.doi.org/10.1590/1414-462×201700030190.

5. Rocha SV, Almeida MMG, Araújo TM, Virtuoso-Junior JS. Prevalência de transtornos mentais comuns entre residentes em áreas urbanas de Feira de Santana, Bahia. Rev Bras Epidemiol. 2010;13(4):630-40. http:// dx.doi.org/10.1590/S1415-790X2010000400008. PMid:21180852.

6. Ludermir AB, Melo Filho DA. Condições de vida e estrutura ocupacional associadas a transtornos mentais comuns. Rev Saude Publica. 2002;36(2):213-21. http://dx.doi.org/10.1590/S0034-89102002000200014. PMid:12045803.

7. Souza MLP. Registro de distúrbios mentais no Sistema de Informação da Atenção Básica do Brasil, 2014. Epidemiol Serv Saude. 2016;25(2):405-10. http://dx.doi.org/10.5123/s1679-49742016000200018. PMid:27869957.

8. Lund C, Breen A, Flisher AJ, Karuma R, Corrigall J, Joska JA, et al. Poverty and common mental disorders in low and middle income countries: A systematic review. Soc Sci Med. 2010;71(3):517-28. http://dx.doi. org/10.1016/j.socscimed.2010.04.027. PMid:20621748.

9. Guimarães MBL, Lima CM, Savi EA, Cardoso E, Valla VV, Stotz EN, et al. Os impasses da pobreza absoluta: a experiência da Ouvidoria Coletiva na região da Leopoldina, Rio de Janeiro (RJ, Brasil). Ciênc. Saúde Coletiva. 2011;16(1):291-300. http://dx.doi.org/10.1590/S1413-81232011000100031.

10. Santos GA, Nunes MO. O cuidado em saúde mental pelos agentes comunitários de saúde: o que aprendem em seu cotidiano de trabalho? Physis. 2014;24(1):105-25. http://dx.doi.org/10.1590/S010373312014000100007.

11. Oliveira AGB, Ataíde IFC, Silva MA, Ataíde IFC, Silva MA. A invisibilidade dos problemas de saúde mental na atenção primária: o trabalho da enfermeira construindo caminhos junto às equipes de saúde da família. Texto Contexto Enferm. 2004;13(4):618-24. http://dx.doi.org/10.1590/S0104-07072004000400015.

12. Souza RC, Scatena MCM. Possibilidades e limites do cuidado dirigido ao doente mental no Programa de Saúde da Família. Rev. Baiana Saúde Pública 2007;31(1):147-160.

13. Nogueira FJS, Brito FMG. Diálogos entre saúde mental e atenção básica: relato de experiência do PetSaúde no município de Parnaíba-PI. Pesqui. Prát. Psicossociais 2017;12(2):374-387.

14. Campos RO, Gama CA, Ferrer AL, Santos DVD, Stefanello S, Trapé TL, et al. Saúde mental na atenção primária à saúde: estudo avaliativo em uma grande cidade brasileira. Ciênc. Saúde Coletiva 2011;16(12):4643-4652. http://dx.doi.org/10.1590/S1413-81232011001300013.

15. Medina MG, Nery Filho A, von Flach P. Políticas de prevenção e cuidado ao usuário de substâncias psicoativas. In Paim JS, Almeida-Filho N. Saúde coletiva: teoria e prática. Rio de Janeiro: MedBook; 2014 p. 479-497.

16. Schneider DR, Lima DS. Implicações dos modelos de atenção à dependência de álcool e outras drogas na rede básica em saúde. PSICO. 2011;42(2):168-78.

17. Silveira PS, Martins LF, Ronzani TM. Moralização sobre o uso de álcool entre agentes comunitários de saúde. Psicol Teor Prat. 2009;11(1):62-75.

18. Oliveira JF, McCallum CA, Costa HO. Representações sociais de Agentes Comunitários de Saúde acerca do consumo de drogas. Rev Esc Enferm USP. 2010;44(3):611-8. http://dx.doi.org/10.1590/S008062342010000300009. PMid:20964035.

19. Alves VS. Modelos de atenção à saúde de usuários de álcool e outras drogas: discursos políticos, saberes e práticas. Cad Saude Publica. 2009;25(11):2309-19. http://dx.doi.org/10.1590/S0102-311X2009001100002. PMid:19936470.

20. Garcia GDV, Silva IF, Cavalcante M, Cervo MR, Zambenedetti G, Zanoti-Jeronymo DV. Apoio matricial na atenção à saúde mental em uma regional de saúde, Paraná, Brasil. Saúde Pesqui. 2017;10(3):423-32. http://dx.doi.org/10.17765/1983-1870.2017v10n3p423-432.

21. Silva CB, Santos JE, Souza RC. Estratégia de apoio em saúde mental aos Agentes Comunitários de Saúde de Salvador - BA. Saude Soc. 2012;21(1):153-60. http://dx.doi.org/10.1590/S0104-12902012000100015. 
22. Machado DKS, Camatta MW. Apoio matricial como ferramenta de articulação entre a saúde mental e a atenção primária à saúde. Cad Saude Colet. 2013;21(2):224-32. http://dx.doi.org/10.1590/S1414$462 X 2013000200018$.

23. Moura RFS, Silva CRC. Silva CRdC. Saúde mental na atenção básica: sentidos atribuídos pelos Agentes Comunitários de Saúde. Psicologia (Cons Fed Psicol). 2015;35(1):199-210. http://dx.doi.org/10.1590/19823703001832013.

24. Silva MAC, Aguiar MGG, Moreira TDS. Entre os nós da rede de saúde mental: as práticas de Agentes Comunitários de Saúde. Rev. Baiana Saúde Pública. 2016;40(3):713-728. https://doi.org/10.22278/23182660.2016.v40.n3.a2185.

25. Instituto Brasileiro de Geografia e Estatística. Censo Demográfico 2010 [Internet]. 2011 [Acesso em: 01 Jul 2018]. Disponível em: http://www.censo2010.ibge.gov.br/

26. Franco FF, Ferla AA. Saúde mental e atenção básica no cuidado aos usuários de álcool e outras drogas. Interface (Botucatu). 2017;21(63):833-44. http://dx.doi.org/10.1590/1807-57622016.0270.

27. Souto MG. Projeto de intervenção para a implantação do novo sistema de informação da atenção básica no município de Itabuna:novo e-SUS AB/SISAB [Trabalho de Conclusão de Curso]. Rio de Janeiro: Universidade Aberta do Brasil, Escola Nacional de Saúde Pública Sérgio Arouca; 2015.

28. Oliveira AEC, Lima IMB, Nascimento JA, Coelho HFC, Santos SR. Implantação do e-SUS AB no Distrito Sanitário IV de João Pessoa (PB): relato de experiência. Saúde Debate. 2016;40(109):212-8. http://dx.doi. org/10.1590/0103-1104201610917. 